\title{
IMPORTÂNCIA DA VELOCIDADE DE CORTE NA OTIMIZAÇÃO DA ETAPA DE POLIMENTO DE ROCHAS ORNAMENTAIS
}

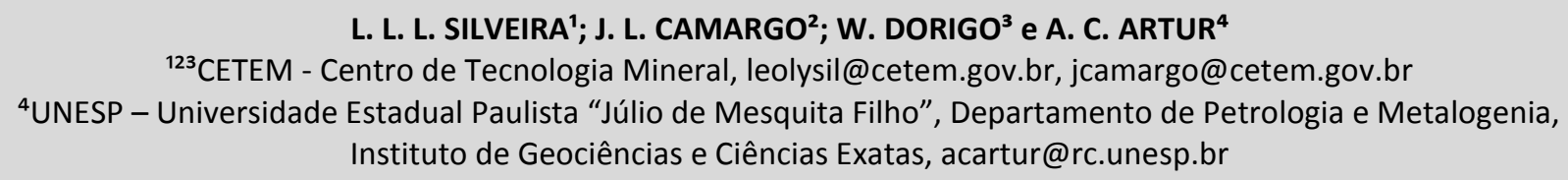

Artigo submetido em novembro/2013 e aceito em janeiro/2014

DOI: http://dx.doi.org/10.15628/holos.2014.1753

\section{RESUMO}

A etapa de polimento de rochas ornamentais ocorre em politrizes, as quais podem ser semi-automáticas ou automáticas. Em ambas as máquinas, a velocidade de rotação do satélite é invariável, e, por consequência, a velocidade de corte, que é a distância percorrida por um rebolo abrasivo em função do tempo, permanece constante. A possibilidade de se alterar a velocidade com que o rebolo abrasivo passa sobre a superfície da chapa de rocha pode permitir uma otimização desse processo. Para esta pesquisa foram definidas 24 situações operacionais distintas, nas quais variou-se a pressão de carregamento (1 e $2 \mathrm{kgf} / \mathrm{cm} 2$ ), velocidade de rotação de satélite $(300,400,500$ e $600 \mathrm{rpm})$ e a exposição do satélite sobre a rocha (1, 2 e 3). Para isso, foi instalado um inversor de frequência em uma politriz semi-automática que possibilitou a variação da velocidade de rotação de satélite para realização dos experimentos. Assim, foram realizados os testes de polimento, sendo cada rocha submetida a 10 sequências de rebolos abrasivos do tipo magnesiano, a saber: 24, 36, 60, 120, 220, 400, 600, 800 1200 e lustro, totalizando 720 amostras. Os resultados mostraram que no sistema tribológico existente no polimento de rochas ornamentais a variável rocha atua como elemento ativo, influenciando no desgaste do rebolo abrasivo e exibindo brilho de acordo com as propriedades petrográficas.

PALAVRAS-CHAVE: polimento, tribologia, brilho

\section{IMPORTANCE OF CUTTING SPEED ON THE OPTIMIZATION OF DIMENSION STONES POLISHING}

\begin{abstract}
The polishing of dimension stones at industrial scale is done in equipments called polishing machines, and they may be semi-automatic or automatic. In both cases the rotation speed of the polishing head is invariable, which implies in a constant cutting speed. The possibility of changing the speed the griding tools pass over the surface of the slab of stone could allow an optimization of this process. For this research 24 distinct operational situations were defined, varying pressure ( 1 to $2 \mathrm{kgf} / \mathrm{cm}^{2}$ ), rotational speed of the polishing head $(300,400,500$ and $600 \mathrm{rpm}$ ) and the exposure time of the polishing head onto the slab of stone $(1,2$, and 3$)$. A frequency inverter
\end{abstract}

was installed in a semi-automatic polishing machine which allowed the variation of the speed of the polishing head for these experiments. The polishing tests were conducted applying, to each kind of stone, 10 sequences of abrasive tools of magnesium type, namely $24,36,60$, $120,220,400,600,800,1200$ and lustrum, totalizing 720 samples. The results showed that the stone is an active element in the tribological system of stones polishing, wearing out more or less abrasives and showing brightness according to the petrographic properties and depending on the solicitation imposed

KEYWORDS: polishing, tribology, brightness. 


\section{INTRODUÇÃO}

O setor de rochas ornamentais tem grande importância para a economia do país, além de ser uma área em crescente desenvolvimento. Segundo dados da Abirochas (2012), as exportações do período janeiro-setembro de 2012 atingiram US\$ 805,31 milhões e 1.700.823,34 t, mantendo variação positiva comparada ao mesmo período de 2011. Apesar do Brasil ainda atuar de forma pouco expressiva no desenvolvimento de pesquisas, nos últimos anos houve uma significativa melhora, em especial no setor de beneficiamento de rochas ornamentais.

O beneficiamento de rochas ornamentais compreende uma sucessão de etapas, que vai desde o desdobramento dos blocos em chapas, conhecido como beneficiamento primário, até o acabamento final, ou beneficiamento secundário, na qual ocorre o polimento. No que diz respeito à qualidade do polimento, Kaschner (1996) descreve que a intensidade do brilho de uma placa de rocha é função das propriedades refletivas dos minerais que a compõem, sendo também inversamente proporcional à rugosidade da superfície. Portanto, a eliminação destas rugosidades herdadas na etapa de serragem e o fechamento dos poros, existentes entre os diferentes minerais que formam a rocha, é que vão imprimindo o brilho e o lustro de cada material. Isto se dá com a utilização de ferramentas abrasivas que, conduzidas em um movimento circular e de atrito sobre o material vão desbastando-o até atingir o grau de polimento desejado, utilizando de abrasivos com granulometrias decrescentes. A qualidade final do polimento ainda encontra-se determinada somente por métodos empíricos. Como regra geral, tal parâmetro é inferido pela granulometria dos abrasivos utilizados durante as etapas de polimento medido com o auxílio de um medidor de brilho, utilizado ainda por poucas empresas do setor (RIBEIRO, et al., 2004). O termo abrasivo pode ser definido como uma partícula ou grão capaz de causar rápido ou eficiente desgaste em uma superfície sólida (STACHOWIAK e BATCHELOR, 1993 apud SILVEIRA, 2008) para o qual há diferentes granulometrias que vão variar em sequência decrescente de acordo com a etapa de desbaste da chapa. Os rebolos abrasivos são ferramentas constituídas por partículas responsáveis pelo corte, imersas em matrizes cerâmicas (cimento Sorel), ou em matrizes poliméricas (poliéster ou epóxi) (FILGUEIRA e AIGUEIRA, 2006), sendo os de matriz cerâmica chamados de abrasivos magnesianos, cuja matriz ligante é composta de óxido de magnésio (MgO) e o elemento abrasivo carbeto de silício $(\mathrm{SiC}$ ) e os resinóides produzidos com liga de poliéster ou epoxídicos e elemento abrasivo o diamante. O processo de polimento de rochas ornamentais está inserido no contexto da Tribologia, que é a ciência que estuda o atrito de superfícies em contato gerado por um movimento relativo (ZUM-GAHR, 1987). Foi primeiramente concebida para a área metal-mecânica, de extrema importância na compreensão dos fenômenos físicos existentes no beneficiamento de rochas ornamentais. O desgaste abrasivo pode ser dividido em relação ao tipo de contato, podendo este ser a dois ou três corpos. O polimento está inserido no contexto tribológico com o desgaste do tipo a dois corpos (rebolo abrasivo e rocha), sendo que o terceiro elemento formador do sistema é a máquina de polir e suas variáveis operacionais. Atualmente, as principais politrizes utilizadas no beneficiamento de rochas ornamentais são classificadas em dois tipos: semi-automáticas e automáticas. Em ambos os casos os rebolos abrasivos são acoplados em cabeçotes, também denominados satélites, no qual, giram em velocidade constante. Acredita-se que a variação desta velocidade pode contribuir de forma a otimizar esta etapa. 


\section{OBJETIVO}

Estudar a resposta que algumas rochas apresentarão quando submetidas a uma nova variável operacional "a variação da velocidade de rotação do satélite de polir", com intuito de otimizar a etapa de polimento de rochas ornamentais.

\section{MATERIAIS E MÉTODOS}

Para estudar a influência da velocidade de rotação de satélite no polimento, foram selecionadas para esta pesquisa três diferentes tipos de rochas ornamentais de aspectos petrográficos, texturais e estruturais distintos, com o objetivo de analisar os resultados em função das características intrínsecas de cada material na ação de desgaste. Além de relacionar o tempo de exposição das chapas ao processo, às velocidades de rotação e a pressão do satélite da politriz. A saber: o gnaisse Preto Indiano (Figura 1A), o granito Cinza Castelo (Figura 1B) e o charnoquito Verde Labrador (Figura 1C). O Cetem/ES adquiriu 6 chapas de rocha bruta por material estudado, num total de $101 \mathrm{~m}^{2}$, para realização dos experimentos.
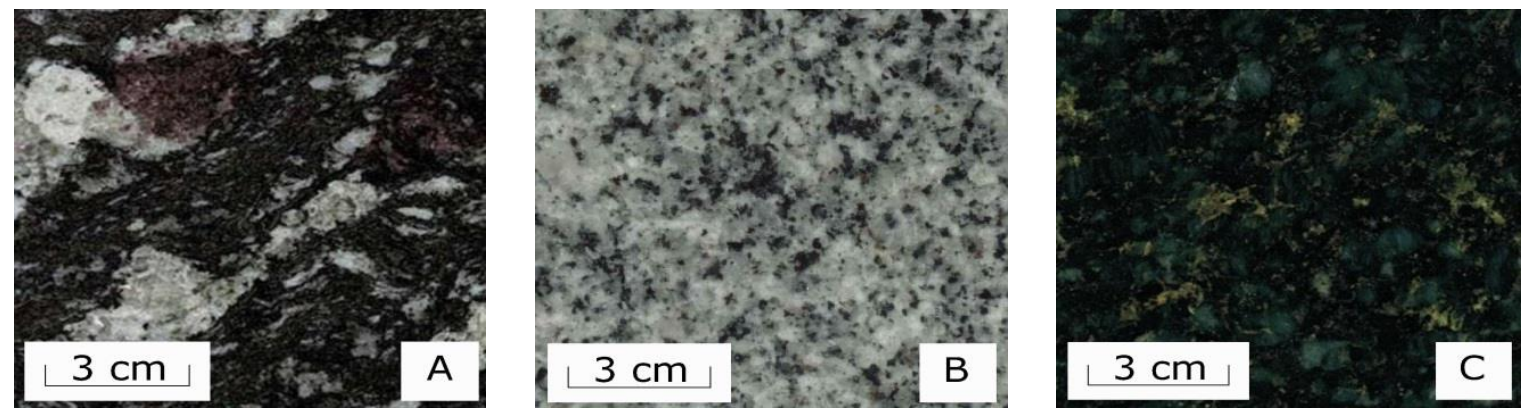

Figura 1. Rochas utilizadas na pesquisa: (A) gnaisse Preto Indiano, (B) monzogranito Cinza Castelo, (C) charnoquito Verde Labrador.

O polimento das rochas citadas foi realizado em uma politriz semi-automática de um cabeçote, da marca Cimef (Figura 2), de propriedade do Laboratório de Beneficiamento de Rochas Ornamentais do Instituto Federal do Espírito Santo (IFES) - Campus Cachoeiro de Itapemirim. Os cabeçotes giram em uma velocidade constante nas politrizes, embora alguns equipamentos atualmente já dispõem de motores controlados por inversores de frequência, não se sabe de fato qual a influência na qualidade do polimento, pois a introdução desse dispositivo nesses equipamentos está relacionada à economia de energia que o mesmo pode proporcionar. Foi instalado um inversor de frequência da marca Delta (Figura 3), modelo VFD007B23, 230 v Trifásico de $1 \mathrm{HP}(0.75 \mathrm{~kW})$, o que possibilitou a variação da velocidade de rotação do satélite em 300,400 , 500 e $600 \mathrm{rpm}$. Em conjunto com duas cargas (1 e $2 \mathrm{kgf} / \mathrm{cm}^{2}$ ) e a exposição ao processo (1, 2 e 3), propiciaram vinte e quatro situações operacionais distintas. 


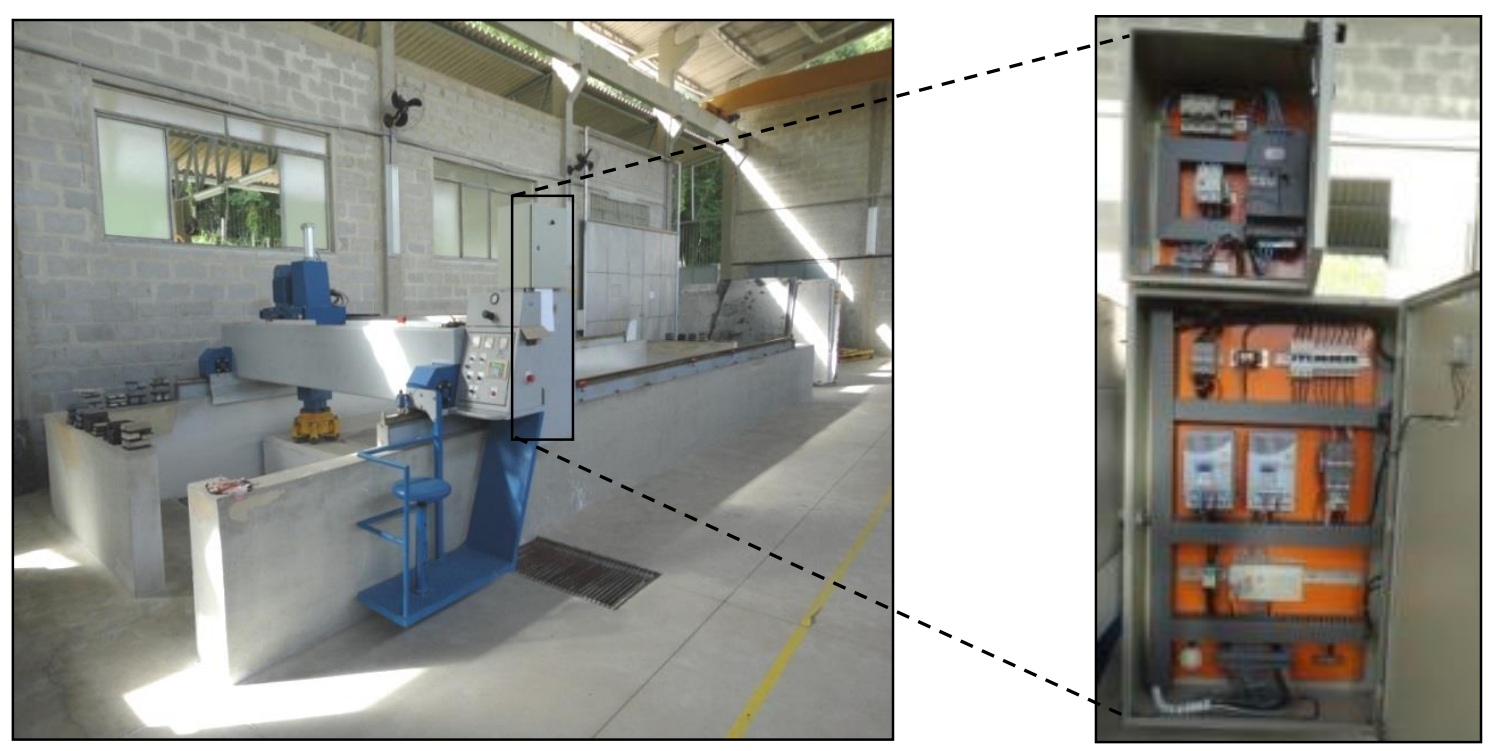

Figura 2. Politriz semi-automática.

Figura 3. Inversor de frequência.

\subsection{Abrasivos Utilizados}

Nesta pesquisa, foram utilizadas dez sequências de abrasivos do tipo magnesianos, que variou de forma decrescente sua granulometria (\#24, \#36, \#60, \#120, \#220, \#400, \#600, \#800, \#1200, Lustro) (Figura 4).

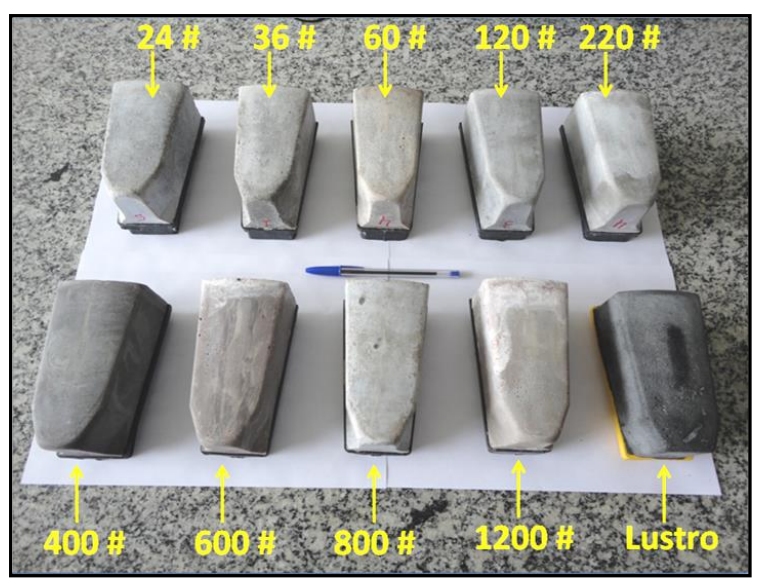

Figura 4. Sequência de abrasivos utilizados.

As chapas foram então submetidas ao polimento (Figura $5 \mathrm{~A}$ ), na qual se variou a carga da politriz, a velocidade de rotação do satélite e a exposição ao processo. Para cada situação operacional foi coletada uma amostra em cada granulometria (Figura 5B), o que totalizou 240 amostras por rocha. Em cada corpo de prova coletado foram realizadas 30 medições de brilho, com o auxílio de um glossmeter modelo micro-tri-gloss da marca Gardner, gerando um total de 7200 medidas por rocha (Figura $5 \mathrm{C}$ ). 

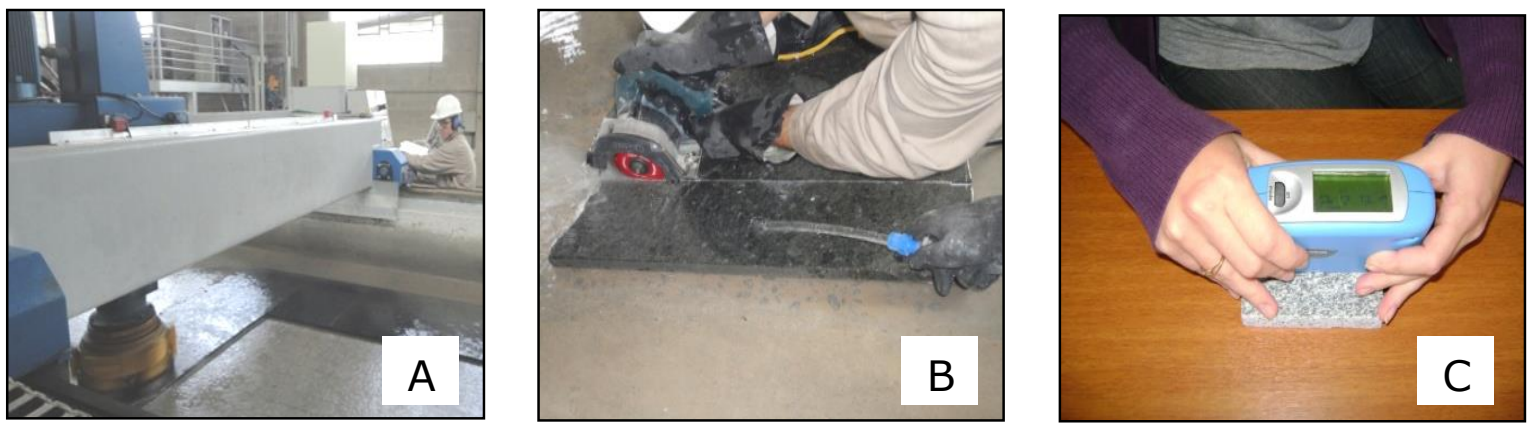

Figura 5. Etapas realizadas na pesquisa. (A) Polimento das chapas; (B) Recorte das amostras; (C) Medição do brilho nos corpos de prova.

Após o polimento, os três diferentes tipos de rochas foram nomeados adotando uma nomenclatura correspondente para cada etapa, de acordo com a figura abaixo:

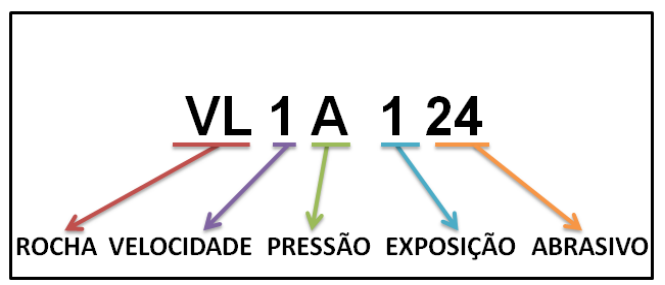

Figura 6. Exemplo da nomenclatura alfa numérica utilizada na pesquisa.

Legenda:

- Rocha: Verde Labrador/VL;

- Velocidade de Rotação do Satélite (RPM): 1=300; 2=400; 3=500; 4=600;

- Pressão de Carregamento do Satélite $\left(K g f / \mathrm{cm}^{2}\right): A=1 ; B=2$;

- Exposição do satélite sobre a rocha: $1 ; 2 ; 3$;

- Abrasivos (Mesh): 24; 36; 60; 120; 220; 400; 600; 800; 1200; Lustro.

\section{APRESENTAÇÃO DOS RESULTADOS}

Na Tabela 1 apresenta os resultados tribológicos de polimento com ênfase no maior brilho obtido com uso de abrasivos da granulometria 120 mesh ao lustro para as três rochas estudadas. 
Tabela 1. Síntese dos resultados tribológicos de polimento.

\begin{tabular}{|c|c|c|c|c|}
\hline $\begin{array}{l}\text { ABRASIVO } \\
\text { (mesh) }\end{array}$ & ROCHA & MAIOR BRILHO & NOMENCLATURA & $\begin{array}{l}\text { VELOCIDADE DE ROTAÇÃO } \\
\text { DE SATÉLITE (rpm) }\end{array}$ \\
\hline \multirow{3}{*}{120} & $\mathrm{VL}$ & 14,80 & 2 B 3 & 400 \\
\hline & $\mathrm{CC}$ & 11,00 & $2 \mathrm{~B} 2$ & 400 \\
\hline & $\mathrm{PI}$ & 18,40 & $1 \mathrm{~B} 3$ & 300 \\
\hline \multirow{3}{*}{220} & $\mathrm{VL}$ & 21,70 & 3 B 3 & 500 \\
\hline & CC & 18,90 & $4 B 3$ & 600 \\
\hline & $\mathrm{PI}$ & 23,30 & $2 \mathrm{~B} 3$ & 400 \\
\hline \multirow{3}{*}{400} & $\mathrm{VL}$ & 40,40 & 2 B 2 & 400 \\
\hline & CC & 35,10 & 3 B 3 & 500 \\
\hline & $\mathrm{PI}$ & 40,10 & $1 \mathrm{~B} 3$ & 300 \\
\hline \multirow{3}{*}{600} & $\mathrm{VL}$ & 50,80 & $2 \mathrm{A3}$ & 400 \\
\hline & $\mathrm{CC}$ & 58,80 & $4 B 3$ & 600 \\
\hline & $\mathrm{PI}$ & 54,40 & $3 \mathrm{~B} 2$ & 500 \\
\hline \multirow{3}{*}{800} & $\mathrm{VL}$ & 63,10 & $1 \mathrm{~B} 3$ & 300 \\
\hline & $\mathrm{CC}$ & 65,60 & $2 B 3$ & 400 \\
\hline & $\mathrm{PI}$ & 67,50 & $2 \mathrm{~B} 2$ & 400 \\
\hline \multirow{3}{*}{1200} & $\mathrm{VL}$ & 71,20 & $4 B 3$ & 600 \\
\hline & $\mathrm{CC}$ & 72,20 & $1 \mathrm{~B} 3$ & 300 \\
\hline & $\mathrm{PI}$ & 77,00 & $3 \mathrm{~B} 3$ & 500 \\
\hline \multirow{3}{*}{ LUSTRO } & $\mathrm{VL}$ & 86,70 & 2 B 2 & 400 \\
\hline & $\mathrm{CC}$ & 84,00 & $1 \mathrm{~A} 3$ & 300 \\
\hline & $\mathrm{PI}$ & 84,80 & 2 B 3 & 400 \\
\hline
\end{tabular}

A análise dos resultados permite afirmar que os maiores valores de brilho foram obtidos em situações operacionais distintas para os três tipos petrográficos testados. Este fato indica que as propriedades petrográficas das rochas constituem os fatores determinantes no controle das demais variáveis operacionais, inclusive influencia no consumo de abrasivos e na obtenção do brilho final. Cabe salientar que a velocidade média de rotação do satélite de polir em politrizes semi-automáticas e automáticas é de 550 rpm.

\section{CONSIDERAÇÕES FINAIS}

De acordo com as informações adquiridas nesta pesquisa constatou-se que o aumento da velocidade com que os abrasivos passam pela superfície da chapa não implica no acréscimo de brilho (Tabela 1). A velocidade de rotação de satélite média/baixa (400/300rpm), na maior parte dos casos, influenciou na obtenção dos melhores resultados de brilho.

Notou-se também que, em geral, os maiores valores de brilho foram alcançados quando foi conjugada a maior pressão do satélite $\left(2 \mathrm{kgf} / \mathrm{cm}^{2}\right) \mathrm{com}$ a exposição máxima ao processo (3). Esta combinação garantiu maior capacidade de desbaste, planificação e fechamento dos poros na superfície da chapa.

\section{REFERÊNCIAS}

1. ASSOCIAÇÃO BRASILEIRA DA INDÚSTRIA DE ROCHAS ORNAMENTAIS (ABIROCHAS). BalançO das exportações e importações brasileiras de rochas ornamentais no período de janeiro a setembro de 2012. São Paulo. Disponível em: <http://www.ivolution.com.br/news/upload_pdf/11360/Informe_13_2012.pdf.>. Acessado em: 10 de novembro de 2012. 
2. FILGUEIRA, M., AIGUEIRA, R. Mecanismos de resistência à abrasão de compósitos à base de Poliéster-SicC para uso em coroas de polimento de Rochas Ornamentais. Polímeros: Ciência e Tecnologia. Vol. 16, n³, p. 187-192, 2006.

3. KASCHNER, D. Tecnologias para beneficiamento. In: ARAÚJO, E.S.; LACERDA FILHO,

4. J.V., FERREIRA, M.C.B.; ARAÚJO, V.A. (orgs.). Anais do II Encontro do Centro-Oeste sobre Granitos, Mármores e Pedras Ornamentais. p. 1-11, 1996.

5. RIBEIRO, R. P. Influências das características petrográficas de granito no processo industrial de desdobramento de blocos. Tese de Doutorado. Universidade de São Paulo. Escola de Engenharia de São Carlos. São Carlos, 205p., 2005.

6. SILVEIRA, L. L. L. Polimento de Rochas Ornamentais: Um Enfoque Tribológico ao Processo. 2008. 203p. Tese (Doutorado) - Escola de Engenharia de São Carlos, Universidade de São Paulo (Brasil).

7. ZUM-GAHR, K. H. Microstructure and wear of materials. Institute of Materials Technology. University of Siegen, Siegen, Federal Republic of Germany, v.10, 358p., 1987 